\title{
BMJ Open Quality Improving patient flow in a regional anaesthesia block room
}

\author{
Brigid Brown, ${ }^{1}$ Ekta Khemani, ${ }^{2}$ Cheng Lin, ${ }^{1}$ Kevin Armstrong ${ }^{1}$
}

To cite: Brown B, Khemani E, Lin C, et al. Improving patient flow in a regional anaesthesia block room. BMJ Open Quality 2019;8:e000346. doi:10.1136/ bmjoq-2018-000346

Received 29 January 2018 Revised 8 August 2018 Accepted 8 March 2019

\section{Check for updates}

(c) Author(s) (or their employer(s)) 2019. Re-use permitted under CC BY-NC. No commercial re-use. See rights and permissions. Published by BMJ.

${ }^{1}$ Department of Anesthesia and Perioperative Medicine, London Health Sciences Centre University Hospital, London, Ontario, Canada

${ }^{2}$ Quality and Risk Management, Humber River Regional Hospital, Toronto, Ontario, Canada

Correspondence to Dr Brigid Brown; brigid.brown@gmail.com

\section{ABSTRACT}

University Hospital is a tertiary academic centre in London, Ontario, Canada. A designated space known as the block room (BR) supports a model of care to perform regional anaesthesia prior to entering the resource intense operating room (OR). Stress due to time pressure was reported by BR staff. It was presumed that upstream delays in patient admission, preparation, transportation and in the BR resulted in late OR starts. There was limited data for a patient's preoperative transit at our institution. A prospective quality improvement project was conceived to understand and address concerns surrounding patient flow. Using Plan-Do-Study-Act (PDSA) methodology, we collected baseline data of patients perioperative transit and performed three PDSA cycles for improvement. We established targets for OR entry time and patient arrival to the BR. We examined communication between the surgical preparation unit, BRandORs, involved stakeholders in decision making and continuously sourced feedback for improvement. Over three incremental rapid PDSA cycles and reaudit of our baseline, we found a statistically significant improvement in patients arriving to the BR $60 \mathrm{~min}$ prior to the scheduled OR time from a baseline of $31 \%-53 \%(p=0.04)$ and patient operations commencing on time improved from $52 \%$ to $65 \%(p=0.03)$. The availability of patients in the BR within 15 min of a decision to have them available reached $98 \%$ from a baseline of $69 \%(p<0.001)$. As a result of the quality improvement process, we were able to significantly improve the flow of the preoperative patient journey at our institution. With a better understanding of complex preoperative processes, we can strategically intervene and potentially improve efficiency, morale and safety.

\section{PROBLEM}

This quality improvement project focused on improving the flow of patients through a regional anaesthesia block room (BR) in London, Ontario, Canada. Optimising patient flow throughout hospitals is important for patient safety and quality of care. ${ }^{1}$ Inefficient patient flow through the BR can result in limited time to effectively perform and test each block, delays in operating room (OR) turnover time, increased costs, increased stress and dissatisfaction for patients and staff. $^{23}$

Prior to this study, no readily available data existed on times for patients' preoperative transit at our institution, and it was not known if a BR visit impacted on OR start times. Medical and nursing staff working in the BR reported feeling stressed by multiple patients requiring blocks at the same time, poor communication between the BR and the ORs and delays in receiving patients from the surgical preparation unit (SPU). Additionally, perceived inefficiencies in the BR was thought to contributed to delays in OR start time.

As part of a continuous model for improvement, this project was undertaken to both understand and address these concerns by collecting baseline data to delineate the issues surrounding flow in the BR and to trial rapid feedback changes in the form of Plan-DoStudy-Act (PDSA) cycles for improvement.

\section{BACKGROUND}

University Hospital (UH) is a tertiary academic centre in London, Canada. There are approximately 10000 surgical cases per year, in the subspecialties of cardiac, urological, plastic, general, oral, otolaryngology orthopaedic and neurosurgeries. Regional anaesthesia and analgesia are offered to many of these surgical patients by a subspecialty group of regional anaesthetists. There are approximately 1900 lower limb arthroplasty surgeries performed annually. In the majority of these cases, a regional anaesthesia procedure is included as part of the surgical anaesthetic and opioid-sparing multimodal analgesic plan.

A designated physical space known as a 'block room', in addition to providing educational and research opportunities, allows the safe and effective performance of regional while optimising OR efficiency. Our BR has been in operation for more than 10 years. Over this time, a number of changes (location, size, case load and staffing) have occurred. Currently, there are five monitored bed areas adjacent to the postanaesthetic room unit staffed by a consultant anaesthetist, an anaesthetic assistant, a nurse and on some days an anaesthetic trainee. A range of regional block types is performed in this space including peripheral nerve blocks of 
the upper and lower limb, truncal blocks and neuraxial techniques. Some of the regional blocks, such as spinal anaesthetics, are time sensitive or time limited and, as such, must be performed as close to the OR start time as possible. Techniques such as peripheral nerve blocks are of longer duration and require longer onset when compared with a spinal anaesthetic. Therefore, running the BR requires precise planning and decision making. Prioritisation of regional anaesthesia blocks is a key component of a BR. With multiple patients ready to receive nerve blocks simultaneously, performing them in a sequence that takes into account the anaesthetic technique (regional alone vs combined), block onset, duration and goal (surgical vs analgesic block) and time to incision and surgical completion is essential for optimal block performance. For this preoperative process to be efficient, there is a need for a 'real time' effective communication strategy, in addition to the expertise of the care teams that prepare patients for the OR.

Improved efficiencies in perioperative care can have significant impact on patient throughput. ${ }^{4}$ The use of a BR has previously been demonstrated to reduce anaesthesia OR time and patient recovery times. ${ }^{5}$ Moreover, the use of regional anaesthesia is effective in reducing postoperative nausea, postoperative pain scores and increasing patient satisfaction. ${ }^{6}$ The process of using the BR at UH involves movement of patients between three physical spaces: (1) the SPU, (2) the BR and (3) the ORs. Once patients are admitted to hospital, they are transferred to the SPU to be checked in for their surgery, clarify the preoperative questionnaire and have preoperative medications administered. Patients are then transferred to the BR for their regional procedure and, after consent is obtained and the regional procedure is performed and tested, they are transferred to the OR for surgery.

In addition to the physical presence of a patient in the $\mathrm{BR}$, there are many variables affecting work flow including the type of block the patient is receiving, the number of ORs requiring blocks and length of time it takes for a patient to arrive from the PSU to the BR.

Inefficient patient flow may limit time to effectively perform and test each block, delay OR turnover, increase costs and reduce satisfaction for patients and staff. ${ }^{7}$ Therefore, studying patient flow in our BR is warranted to maximise efficiency and patient satisfaction.

Using patient data to model the impact of parallel processing on OR efficiency has shown to be potentially beneficial if regional anaesthesia is performed outside of the OR. ${ }^{9}$ Improving patient flow has been discussed in multiple areas of the hospital system, particularly in the emergency departments and ward discharge planning. ${ }^{10} 11$ In the OR environment, patient flow has also been studied in the preadmission clinic setting. ${ }^{12}$ Implementing a regional anaesthesia BR has also been shown to enhance efficiency and flow. ${ }^{13}$ However, to our knowledge, this is the first quality improvement study using the PDSA methodology for the evaluation of patient flow within an existing regional anaesthesia BR.

\section{MEASUREMENT}

This project was conducted from September 2016 to June 2017.

The aims of our study were to have: (1) $90 \%$ of patients arrive to the BR within 60 min of their OR start time, (2) $90 \%$ of patients arrive to the BR within 15 min of calling the SPU and (3) $90 \%$ of patients arrive to the OR within 10 min of their scheduled start time.

Our goals were determined as part of the project design in consultation with the regional anaesthesia team at our institution. Arrival time to the BR was calculated from previous block performance times. On average, the time required to consent a patient, prepare and perform a regional block on a patient is approximately $45-60 \mathrm{~min}$, especially when allowing for time to teach an anaesthesia trainee. This time is consistent with a recent finding that time to readiness for surgery in a BR was $44 \mathrm{~min} .{ }^{13}$ The choice of a $60 \mathrm{~min}$ goal includes outlining the planned block along with risks and benefits to the patient, ensuring intravenous access and monitoring are in place, the operative site has been marked, sedation is administered, the block is performed and the desired sensory block has been tested to be complete prior to transfer to the OR. If a patient arrives to the BR less than $60 \mathrm{~min}$ prior to their scheduled surgical start time, this process would be rushed or incomplete, or the patient may be late arriving to the OR.

The second goal of efficient communication and subsequent transfer of patients from SPU to BR was set as $15 \mathrm{~min}$. There was concern prior to undertaking this study that the SPU was underserviced with staff and bed numbers to prep all of the surgical patients and that this goal would be challenging to meet. If the patient was ready before a request was made by the BR to SPU, an orderly was paged to complete the transfer. If the orderly was not readily available, there was a potential delay in the patient's arrival to the BR, and the first goal of a $60 \mathrm{~min}$ timeline in the BR would not be achieved.

The time at which operating rooms commence a new surgical case is known as 'the scheduled OR start time'. This time is tracked and reported on. When a delay does occur, the reason(s) is/are documented. Having patients arrive to the OR within $10 \mathrm{~min}$ of their scheduled start time is an indication of global OR efficiency of which the $\mathrm{BR}$ is a part. For the first case of the day, measuring this goal suggests that the preoperative phase of the patient journey is managed in a time efficient manner. With regard to the last case on an OR list, failure to commence within $30 \mathrm{~min}$ of the scheduled time can lead to cancellation of the case or the case running beyond the scheduled end time. Each of these outcomes has social and financial implications.

Baseline data were collected from the 1st to 30th of September 2016 and 2-4weeks' worth of data collected following implementation of each patient flow intervention strategy. A repeat baseline data measurement was collected 1 month after the PSDA cycles were complete to assess the sustainability in our new practice. 
Times of the patient journey were recorded including time of admission to hospital, time of admission to and leaving the SPU, time of arrival and discharge from the $\mathrm{BR}$, time of starting and finishing regional procedure and time of arrival to the OR, and OR start time. The duration that patients spent in the SPU or BR and travelling between each location were calculated using the above data.

Also noted were the times the OR called the BR to inform them to proceed with the next block, and the time the BR then contacted the SPU to receive the patient. The total number of patients accessing the BR, the planned surgery and types of blocks performed were also recorded.

Our baseline data demonstrated that we were servicing an average of ten patients a day in the BR, representing approximately $15 \%-20 \%$ of total cases in the OR complex. On average, $28 \%$ of procedures occurred at the start of the operating day when multiple ORs start at the same time, and all ORs needed patients with completed blocks simultaneously.

During this period, $51 \%$ of patients were arriving to the OR more than $10 \mathrm{~min}$ after their scheduled OR start time. In $28 \%$ of cases, the OR failed to notify the BR they were ready for the next patient to have their block placed. Also noted was that $25 \%$ of patients took longer than $15 \mathrm{~min}$ to arrive to the BR from SPU once a request was made for transfer. These results were the driving force behind the development of this quality improvement project to improve flow.

\section{DESIGN}

Following the collection of our baseline information over 4 weeks, it was clear there were opportunities and drives for improvement in patient flow during the perioperative period when using the BR. We began our interventions by presenting the findings from our baseline data to stakeholders in the BR, SPU, the OR and administration and encouraging feedback and suggestions for improvement in an audit and feedback cycle.

Overarching themes from our baseline information and from staff feedback included improving communication between the BR, the SPU and the OR as well as the need to arrange for earlier patient arrival into the BR to ensure adequate time to obtain consent and to complete and test each block prior to surgery.

We secured a 'champion' for this project from each stakeholder group to promote support during our rapid cycle changes.

Using baseline data and feedback received from key stakeholders, we implemented PDSA methodology to complete our quality improvement strategy over an 8-month period.

A $\mathrm{X}^{2}$ test for independence was used to statistically analyse and compare our targets from our baseline to our subsequent PDSA cycles.
Table 1 An overview of the study design with specific changes in each PDSA cycle listed

\begin{tabular}{|c|c|}
\hline PDSA cycle & Changes \\
\hline PDSA 1 & $\begin{array}{l}\text { Feedback meetings with stakeholders (below) } \\
\text { presenting baseline data and sourcing input } \\
\text { and suggestions: } \\
\text { Nurse education meeting. } \\
\text { Block room and recovery staff meeting. } \\
\text { Anaesthetists' weekly journal meeting }\end{array}$ \\
\hline \multirow[t]{4}{*}{ PDSA 2} & $\begin{array}{l}\text { Change of physical location of surgical prep } \\
\text { for patients scheduled for regional anaesthesia } \\
\text { from the general surgery prep area to within } \\
\text { the block room. }\end{array}$ \\
\hline & $\begin{array}{l}\text { Allocating a prep nurse to prep the patients in } \\
\text { the block room. }\end{array}$ \\
\hline & $\begin{array}{l}\text { Concurrent prep of patients with block room } \\
\text { staff consent, intravenous insertion and } \\
\text { labwork check. }\end{array}$ \\
\hline & $\begin{array}{l}\text { Highlighting patients scheduled for regional } \\
\text { anaesthesia the day before through } \\
\text { stakeholder email and a note on the patient's } \\
\text { electronic and physical file flagging patient to } \\
\text { be prepped in the block room. }\end{array}$ \\
\hline \multirow[t]{4}{*}{ PDSA 3} & $\begin{array}{l}\text { Email communication of planned } \\
\text { communication loop to surgical and } \\
\text { anaesthesia teams. }\end{array}$ \\
\hline & $\begin{array}{l}\text { In person explanation of communication loop } \\
\text { to nursing staff in the OR. }\end{array}$ \\
\hline & $\begin{array}{l}\text { Block room staff highlighting the } \\
\text { communication loop in phone calls to the OR } \\
\text { checking on progress of the previous patient. }\end{array}$ \\
\hline & $\begin{array}{l}\text { Consistent nursing staff working in the block } \\
\text { room of nurses who indicated interest in } \\
\text { regional anaesthesia rather than previous } \\
\text { random allocation to the block room. }\end{array}$ \\
\hline
\end{tabular}

OR, operating room; PDSA, Plan-Do-Study-Act.

\section{STRATEGY}

We undertook three PDSA test cycles over a 6 month period (table 1 provides a summary of these details).

\section{PDSA cycle 1}

An audit and feedback loop, with baseline data of times of preoperative transit collected over 4 weeks and presented to stakeholders to source feedback and buy-in to the planned interventions. In this first PDSA cycle, we found no change in our baseline data or improvement in our study goals following these presentations; however, this cycle provided us with invaluable information and ideas for the further PDSA cycles.

\section{PDSA cycle 2}

To address the issue of delays in patient transfer from the SPU to the BR, we began to prepare those patients expected to receive a block in the BR instead of in the SPU, which is located in a different physical location and a significant distance away from the BR and OR. Patients 
who were identified to undergo a regional procedure were flagged the day before their surgery by BR staff, and an email with this information was sent to admissions, SPU and the BR team. An SPU nurse was allocated to the $\mathrm{BR}$, which allowed these flagged patients to arrive to the BR directly from the admissions desk. They were then prepared in the BR. By preparing patients for their block and surgery in the BR and removing the step of transporting patients down the hall from the SPU location, we allowed an earlier discussion of the planned regional procedure between the BR anaesthetist and the patients and an earlier commencement of the block.

\section{PDSA cycle 3}

A communication loop was developed to allow more effective communication and timely block commencement. As soon as a patient left the BR for surgery, the next patient on the operating list was immediately identified and preparations (if not already underway) started. This improved the chance of having sufficient time for consent, ensuring all administrative requirements were complete and performing the regional block(s) required for surgery. The OR was given the responsibility to call the BR when they estimated approximately 30 min of surgery remained. This was a cue to complete all blocks including spinals on their next patient. This allowed the BR team to determine priority, position the patient, complete sonoanatomy and perform the intended blocks. To complete this communication loop and ensure a phone call had not been missed, if the BR had not heard from the OR by 30 min before the next scheduled surgery, the BR would call the OR to confirm timing to plan for the next case.

\section{RESULTS}

Figure 1 shows the results of our first goal that $90 \%$ of patients entering the BR 60 min prior to scheduled start time. Our baseline data showed only $31 \%$ of patients were arriving $60 \mathrm{~min}$ before their scheduled start time.
Our interventions improved this value to $53 \%$, a statistically significant improvement $(\mathrm{p}=0.04)$ although still not achieving our goal of $90 \%$.

Figure 2 shows the results of our second goal to have $90 \%$ of patients arrive to BR within 15 min of calling the SPU. At baseline, $69 \%$ of patients were arriving within this time, and by the end of our PDSA cycles, we improved this value to $98 \%$, a statistically significant improvement $(\mathrm{p}<0.001)$.

Figure 3 outlines our third study goal of $90 \%$ of patients arriving to the OR within $10 \mathrm{~min}$ of their scheduled start. Baseline data showed this occurred in 52\% of cases. Following our interventions, there was a statistically significant improvement to $65 \%(\mathrm{p}=0.03)$, although still not reaching our target of $90 \%$.

Figure 4 is a flow diagram of the patient journey throughout our hospital as well as communication pathways. The PDSA cycle changes we implemented in this project are also highlighted in this figure.

Figure 5 is a flow diagram illustrating the original patient journey before prior to the commencement of this project and as well as planned future pathways.

\section{LESSONS AND LIMITATIONS}

One of the biggest limitations we experienced is the significant variability within each day and between days that contributes to the peaks and troughs of work flow in our BR. Some of these variables include: (1) the number of patients using the BR each day, (2) the medical comorbidities of patients altering the time required for surgical preparation and anaesthesia consultation, (3) the type of surgical procedure and block(s) offered, (4) staffing in the SPU, the BR and recovery room, (5) the number of surgeries running ahead or behind scheduled time, (6) patients arriving late to the hospital and (7) the clustering of cases with a similar start time. This provided a challenge, as busy days were more affected by patient flow 


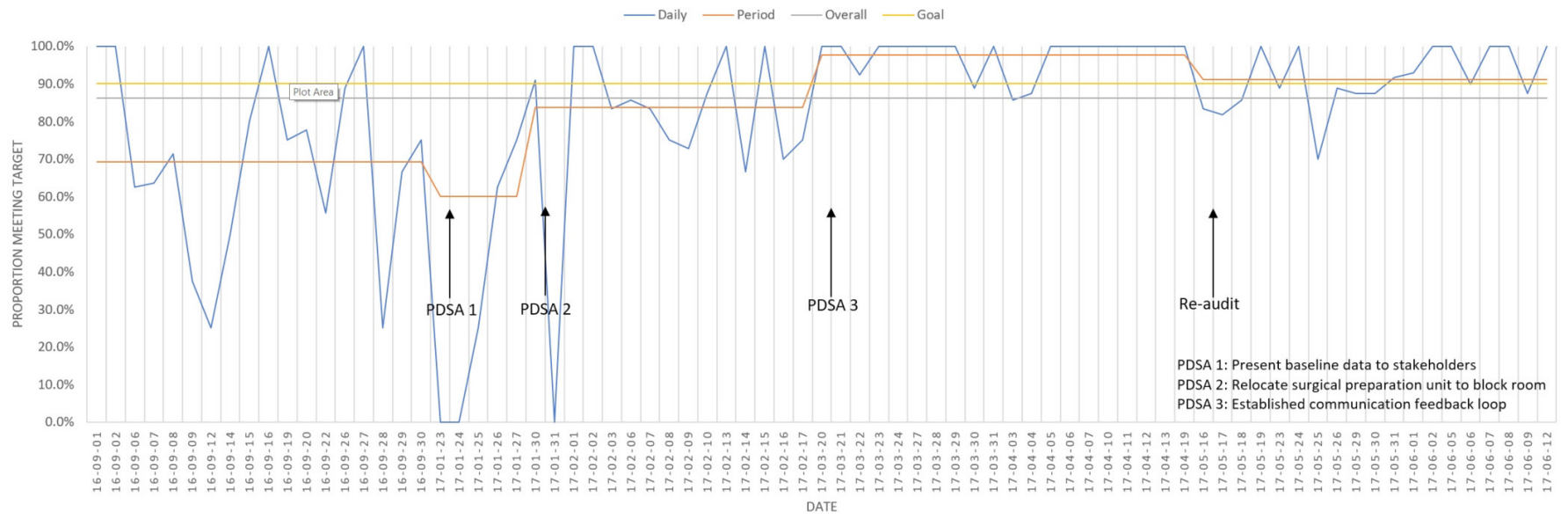

Figure 2 Run chart 2: the percentage of patients entering the block room within 15 min of calling the surgical preparation unit. This improved from $69 \%$ to $98 \%(p<0.001)$. PDSA, Plan-Do-Study-Act.

inefficiency than the slower days. We continue to address this by highlighting expected busier days in the daily email sent to all staff the day before surgery, generating a priority list for patient preparation to ensure patients requiring a more time-demanding block are prepped before patients having a spinal and requesting extra help for the start of the day from the anaesthetists scheduled to the patient's OR to complete time-dependent neuraxial anaesthesia. With regard to variation in OR scheduled time, we continue to work towards effective communication so that planning and prioritisation is appropriate.

Collection of data was also a challenge in this project. While most of the information required for analysis of our system was available from the electronic patient information system, manual data collection was still necessary. This was yet another paper form for nurses to complete in their already paperwork heavy role. When the BR was at its busiest, it could be time consuming to ensure the data collection form was completed. Our limitation with IT resources during the study did add work for the study team as described and slowed some of our PDSA cycles. However, our data from this project showing improved patient flow allowed us recognise and to advocate for an improvement in IT resources with the hospital administration. Since the completion of this study, enhanced communication tools have been added to the electronic perioperative tracking board. This allows staff in the SPU, the BR, the ORs and recovery room to enter data and view information about each patient. With these improvements in IT, tracking of patient movement and communication between prep, the BR and the OR has also been improved.

During this project, modifications to the BR that we did not plan but occurred as a part of larger system and provincial changes. We noticed an increase in patients admitted to the BR, many of which required neuraxial anaesthesia (up to an average of thirteen blocks per day and up to six blocks were being completed for the first cases of the day). To aid these new processes, workforce issues were addressed with an additional nurse was

PROPORTION OF PATIENTS ENTRING OR ON TIME

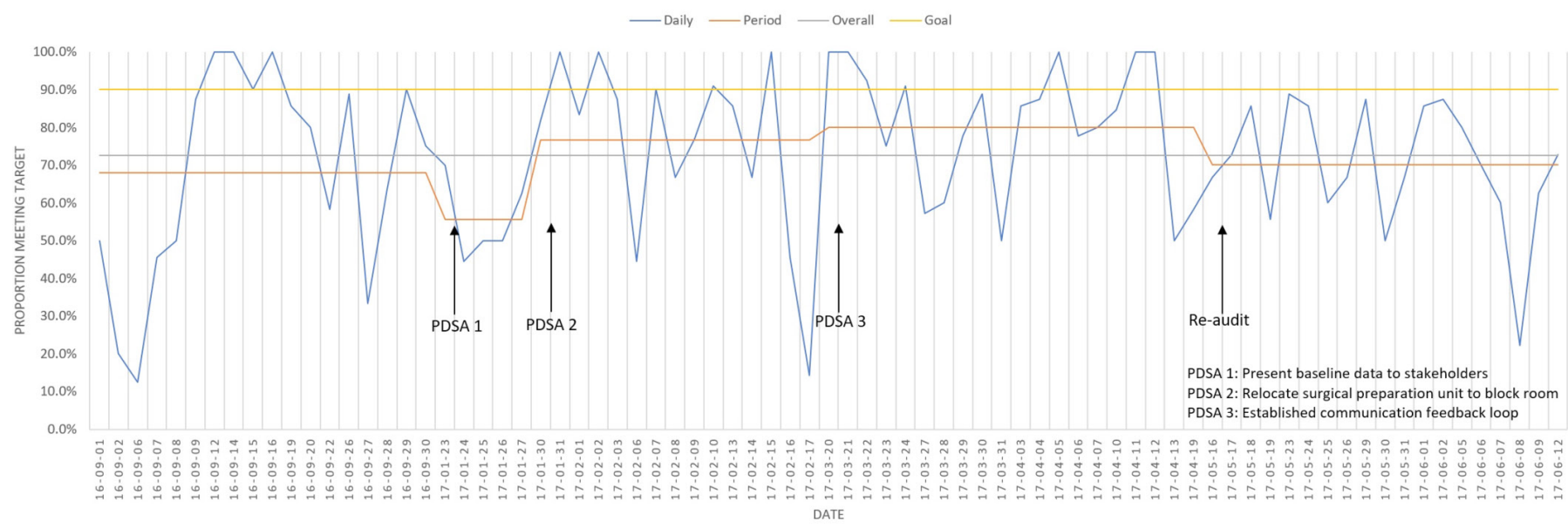

Figure 3 Run chart 3: the percentage of patients arriving to the operating room within 10 min of their scheduled start which improved from $52 \%$ at baseline measurement to $65 \%(p=0.03)$. PDSA, Plan-Do-Study-Act. 


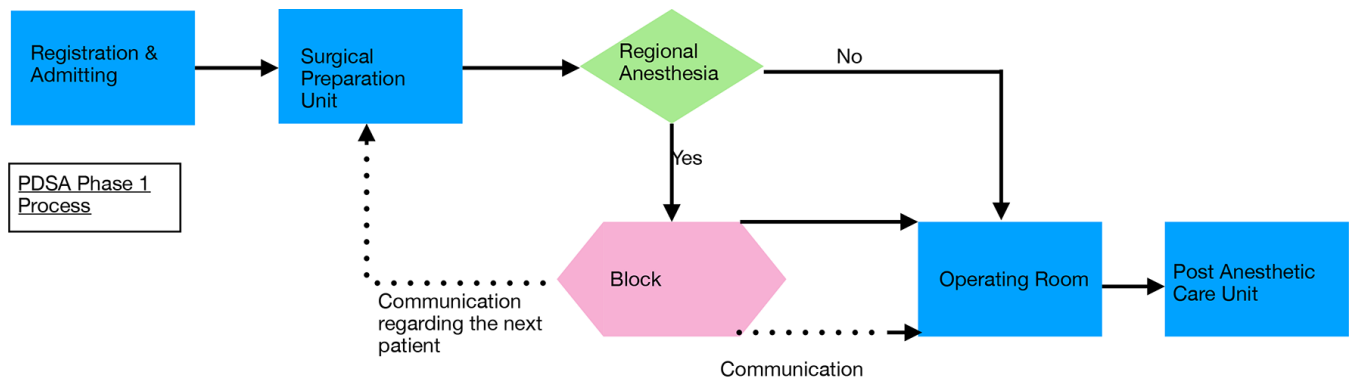

assigned to work in the BR to ensure patient monitoring and safety, although there was no increase in medical staff. These changes to the BR reflected its importance in the larger hospital and provincial system and demonstrated an even greater need to continue to work towards enhancing patient flow and efficiency without compromising safety.

The BR studied in this project is part of the perioperative process in a Canadian academic tertiary health sciences centre. The Canadian healthcare system is a publicly funded system with no private option. Most surgical procedures that use the BR are subject to wait lists. In addition to clinical responsibilities, there is a mandate for both research and educational activity. Any improvements in the quality of care provided and the efficiency of patient flow through the BR results in a better work environment. Additional benefits to efficiency can contribute to financial gains through less overtime paid to OR staff. An efficient BR may contribute to a social benefit of more procedures performed in a single day or on a single operating list; however, this requires a broader approach to a very complex issue. The specifics of our QI project may have limited application to other institutions as each institution faces its own challenges from variations in staffing, space, equipment, expertise, scheduling, communication, patient volumes and guidelines. However, as institutions evaluate perioperative processes to improve patient care through the introduction of a BR, some of the lessons learnt here may be of value.

\section{CONCLUSION}

This quality improvement project aimed to understand and enhance patient flow through a regional anaesthesia $\mathrm{BR}$ while ensuring that patient-centred care was timely, efficient and safe. Central to this improvement is better communication between perioperative areas, and a streamlined patient transfer process to increase efficiency in the patient journey.

We have made statistically significant improvements in all three project goals with resultant better flow in this preoperative journey. There was an increase in the percentage of patients arriving to the $\mathrm{BR} 60 \mathrm{~min}$ prior to scheduled operating start time from $31 \%$ to $53 \%$ $(\mathrm{p}=0.04)$, being present in the BR within $15 \mathrm{~min}$ of making a decision to have them be in the BR from $69 \%$ to $98 \%(\mathrm{p}<0.001)$ and arriving to the OR within $10 \mathrm{~min}$ of their scheduled start time from $52 \%$ to $65 \%(\mathrm{p}=0.03)$.

We believe the changes we have made are sustainable in our institution as remeasuring our baseline data at 10 months showed sustainable improvement. This has helped in maintaining support and acceptance from staff. The changes we have implemented will be able to continue long term, particularly the decision to prep patients in the BR rather than the overcrowded SPU. Also, using consistent, invested nursing staff in the BR rather than different people unfamiliar with the BR operations increases the efficiency of preparing patients and equipment for regional anaesthesia. By demonstrating the benefits and improved efficiency that the BR provides for patients as we have done in this study, a case can be made for improved staffing (there are now two BR nurses assigned to the BR), IT support (as we have done since the conclusion of this study) and physical space (there are now six monitored bays within the BR). Improved efficiency allows stakeholders to advocate for maintenance and improvements to the BR.

Future directions, as outlined in figure 5, include advancing the newly implemented communication loop 

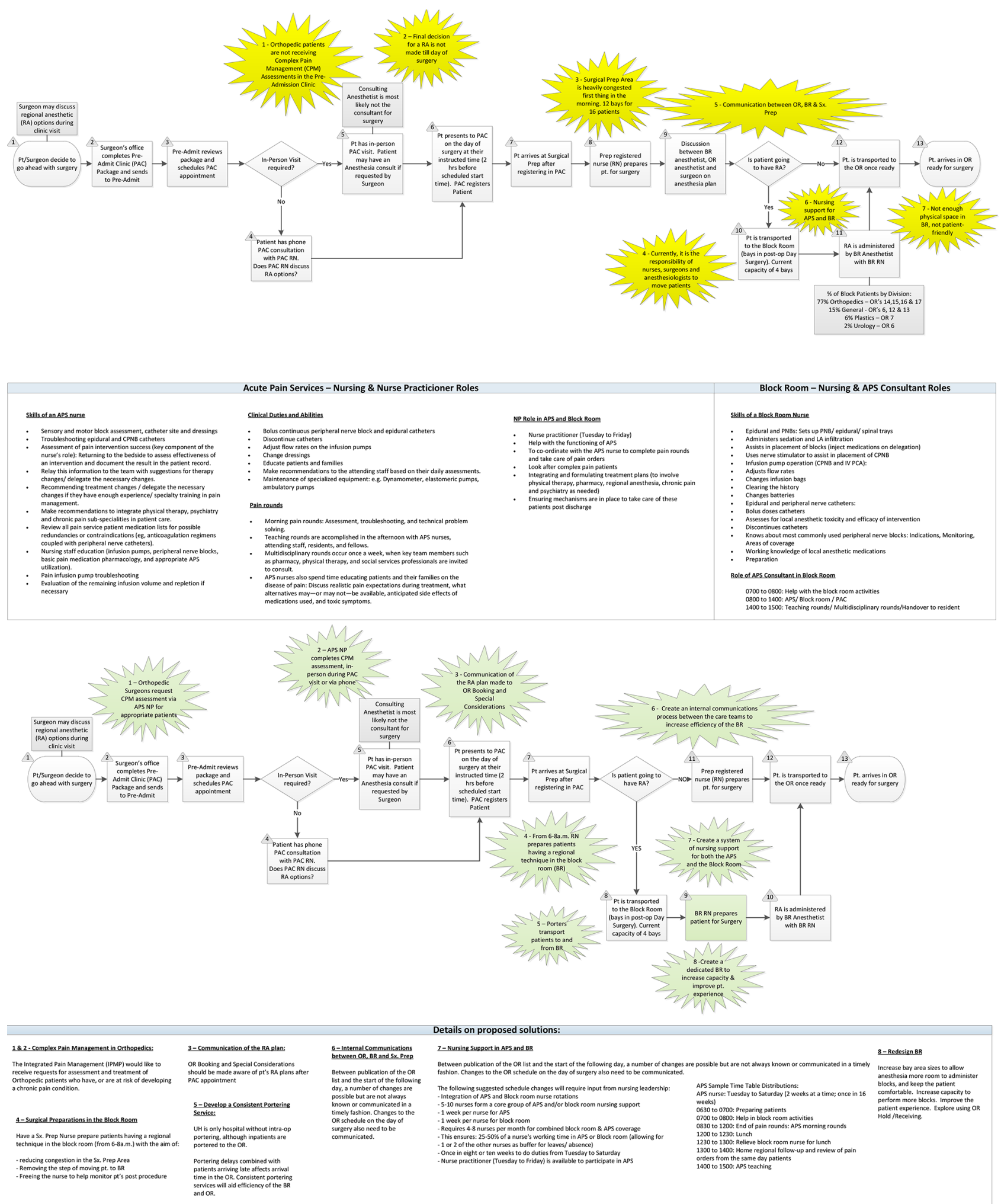

Figure 5 Flow diagram illustrating the original patient journey before this project and future pathways. BR, block room; OR, operating room; UH, University Hospital.

by taking advantage of a computer-based patient tracking system already in use at our hospital. By expanding electronic communication options such as 'prepare patient' by the OR staff, or 'block completed' by BR and OR staff within the patient tracking system, we believe this will allow real-time, important up-to-date information about the timing of patient readiness to be conveyed without an interrupting phone call. We are also planning to add 
information to the daily BR plan email distributed to staff to highlight patients to be prepared first at the start of the day to improve the early morning flow. For instance, requesting a patient having a femoral nerve catheter to be prepped first as this takes longer to insert and can be placed earlier before surgery with less time sensitivity than a neuraxial procedure. We also plan to evaluate the time patients arrive to the hospital and develop an accelerated pathway to ensure they are admitted first to facilitate the additional time required in the BR.

Overall, efficient patient flow is essential to patient safety and high-quality patient-centred care. Rushing due to time and situational stress can result in suboptimal care. Team work and effective communication can improve patient flow, the patient experience and provider satisfaction and performance in a complex perioperative environment. The results of our quality improvement project have highlighted a plan for a continuous model for improvement where we plan to use this work to highlight areas for improvement and to advocate for time, as well as personnel and system resources, which are required to improve this dynamic BR efficiency process.

Contributors BB: overall project design, development of the data collection sheet, the creation of the PDSA cycles, facilitator of stakeholder meetings for feedback and discussion and primary manuscript writing. EK: initial project design contributor and manuscript writing. CL: project design contributor, data collection, project statistics, manuscript writing and editing. KA: project supervisor and mentor, originally developed the concept of evaluating patient flow, assistance in facilitating stakeholder meetings and manuscript writing and editing.

Funding The authors have not declared a specific grant for this research from any funding agency in the public, commercial or not-for-profit sectors.

Competing interests None declared.

Patient consent for publication Not required.

Provenance and peer review Not commissioned; externally peer reviewed.
Open access This is an open access article distributed in accordance with the Creative Commons Attribution Non Commercial (CC BY-NC 4.0) license, which permits others to distribute, remix, adapt, build upon this work non-commercially, and license their derivative works on different terms, provided the original work is properly cited, appropriate credit is given, any changes made indicated, and the use is non-commercial. See: http://creativecommons.org/licenses/by-nc/4.0/.

\section{REFERENCES}

1. Institute for Healthcare Improvement. Optimizing patient flow: moving patients smoothly through acute care settings. IHI Innovation Series white paper. Boston: Institute for Healthcare Improvement, 2003.

2. McHugh M, Van Dyke K, McClelland M, et al. Improving Patient Flow and Reducing Emergency Department Crowding; a Guide for Hospitals AHRQ Publication no 11(12)-0094. 2011.

3. Hostetter M, Klein S. In focus: improving patient flow - in and out of hospitals and beyond. Quality Matters Archive 2013.

4. Saleh KJ, Novicoff WM, Rion D, et al. Operating-room throughput: strategies for improvement. J Bone Joint Surg Am 2009;91:2028-39.

5. Armstrong KP, Cherry RA. Brachial plexus anesthesia compared to general anesthesia when a block room is available. Can $J$ Anaesth 2004;51:41-4.

6. Liu SS, Strodtbeck WM, Richman JM, et al. A comparison of regional versus general anesthesia for ambulatory anesthesia: a meta-analysis of randomized controlled trials. Anesth Analg 2005;101:1634-42.

7. Garrett S. Improving patient flow: how two trusts focused on flow to improve the quality of care and use available capacity effectively: The Health Foundation, 2013.

8. Wilson M, Siegel B, Williams M. Perfecting patient flow. Washington DC: National Association of Public Hospitals and Health Systems, 2005.

9. Brown MJ, Subramanian A, Curry TB, et al. Improving operating room productivity via parallel anesthesia processing. Int $\mathrm{J}$ Health Care Qual Assur 2014;27:697-706.

10. Evans B, Potvin C, Johnson G, et al. Enhancing patient flow in an acute care hospital: successful strategies at the Juravinski Hospital. Healthc Q 2011;14:66-74.

11. Chartier L, Josephson $\mathrm{T}$, Bates $\mathrm{K}$, et al. Improving emergency department flow through Rapid Medical Evaluation unit. BMJ Qual Improv Rep 2015;4:u206156.w2663.

12. Stark C, Gent A, Kirkland L. Improving patient flow in pre-operative assessment. BMJ Qual Improv Rep 2015;4:u201341.w1226.

13. Chazapis M, Kaur N, Kamming D. Improving the peri-operative care of patients by instituting a 'Block Room' for Regional Anaesthesia. BMJ Qual Improv Rep 2014;3:u204061.w1769. 